\title{
Percepção da qualidade de vida em atletas de atletismo e natação paralímpica
}

\author{
Gabriel Lucas Morais Freire ${ }^{a}$ (D), Carla Thamires Laranjeira Granja ${ }^{b}$ (D), \\ Vanthauze Marques Freire Torres ${ }^{b}$ (D), Gustavo César de Vasconcelos ${ }^{c}$ (i), \\ Milena Pedro de Morais ${ }^{\mathrm{d}}$

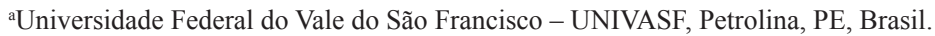

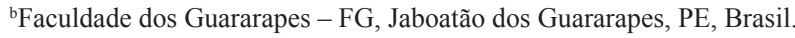 \\ 'Universidade Federal de Pernambuco - UFPE, Recife, PE, Brasil. \\ ¿Universidade São Judas Tadeu - USJT, São Paulo, SP, Brasil.
}

\begin{abstract}
Resumo: O objetivo do presente estudo foi avaliar a percepção da qualidade de vida (QV) dos atletas paralímpicos das modalidades de atletismo e natação. Tratou-se de um estudo descritivo e de corte transversal com 32 paratletas, sendo 22 de atletismo e 10 de natação. Para avaliação da QV foi utilizado o questionário de avaliação abreviado da qualidade de vida da Organização Mundial da Saúde. Para análise dos dados foi utilizado o teste de Shapiro-Wilk; como os dados apresentaram distribuição não paramétrica, foi adotada a apresentação descritiva em mediana (Md) e intervalo interquartílico (Q1; Q3). Para comparar a QV em função do gênero e do patrocínio, foi utilizado o teste de "U" de Mann-Whitney, e para comparar a QV entre o nível de escolaridade, foi utilizado o teste de Kruskal-Wallis. Observou-se entre os domínios de QV que o domínio meio ambiente obteve o menor índice e o domínio social obteve o maior índice entre os paratletas pesquisados. Em relação aos subgrupos mais prejudicados os menores valores encontrados foram entre os paratletas com níveis de escolaridades fundamental I e nível superior, que apresentaram diferenças nos domínios ambientais e de QV geral, fundamental I com ensino médio, apenas na QV geral e fundamental II com ensino superior, apenas no domínio ambiental. Não foram constatadas diferenças significativas na percepção da QV entre os paratletas com e sem patrocínio, e entre as modalidades atletismo e natação. Conclui-se que os atletas paralímpicos que possuem menor nível de escolaridade apresentaram-se como os grupos com menores percepções de QV.
\end{abstract}

Palavras-chave: Qualidade de Vida, Atletismo, Natação, Atletas Paralímpicos.

\section{Perception of quality of life in athletes of athletics and swimming paralympic}

\begin{abstract}
The objective of the present study was to evaluate the perception of the quality of life (QoL) of Paralympic athletes of athletics and swimming. It was a descriptive and cross-sectional study with 32 Paralympic athlete. To evaluate the QoL, the questionnaire of abbreviated quality of life evaluation of the World Health Organization was used. For the analysis of the data the Shapiro-Wilk test was used; because the data presented non-parametric distribution, the descriptive presentation was adopted in median (Md) and interquartile range (Q1; Q3). To compare QoL according to gender and sponsorship, the Mann-Whitney "U” test was used, and the Kruskal-Wallis test was used to compare the QoL between the level of schooling. There was a lower perception of QoL in the environmental domain and a greater perception in the social domain among the parathletes surveyed. In relation to the most impaired subgroups, the lowest values were found among paratrans with primary and higher levels of schooling presented differences in environmental domains and general QoL, fundamental I with secondary education, only in general and fundamental QoL II with higher education, only in the environmental domain. There were no significant differences in the perception of QoL between Paralympic athlete with and without sponsorship, and between athletics and swimming modalities. It is concluded that Paralympic athletes who have lower level of schooling presented themselves as the groups with lower perceptions of QoL.
\end{abstract}

Keywords: Quality of Life, Athletics, Swimming, Paralympic Athletes.

Autor para correspondência: Gabriel Lucas Morais Freire, Universidade Federal do Vale do São Francisco, Rua Arnóbio Marquês, 310 , Santo Amaro, CEP 50100-130, Recife, PE, Brasil, e-mail: bi88el@gmail.com

Recebido em Ago. 22, 2018; 1 ${ }^{\text {a }}$ Revisão em Dez. 13, 2018; 2 ${ }^{\text {a }}$ Revisão em Jan. 29, 2019; Aceito em Jan. $31,2019$. 


\section{Introdução}

O esporte paralímpico, com o passar dos anos, vem se tornando um fenômeno esportivo relevante, colocando as Paralimpíadas como o terceiro maior evento do mundo, ficando atrás apenas das Olimpíadas e da Copa do Mundo (BECKMAN et al., 2014). Desta forma, essa área tem despertado o interesse de pesquisadores nas áreas da ciência do esporte, buscando compreender a influência do esporte de alto rendimento na vida dos paratletas, no sentido das capacidades funcionais e dos domínios sociais (INTERNATIONAL..., 2018; BARYAEV; SHELKOV; EVSEEV, 2009).

Nos últimos anos, paratletas brasileiros têm alcançado resultados expressivos, em especial nas últimas três ediçôes dos Jogos Paralímpicos, nas quais a Comissão Brasileira se configurou entre as 10 melhores seleçóes paralímpicas do mundo (INTERNATIONAL..., 2018). Desde a sua primeira participação nos Jogos Paralímpicos em 1972, a Comissão Brasileira conquistou 301 medalhas, sendo 87 de ouro (INTERNATIONAL..., 2018).

É importante destacar que, do total de medalhas conquistadas pelo Brasil nos Jogos Paralímpicos, aproximadamente $50 \%$ delas pertencem ao atletismo e natação (INTERNATIONAL..., 2018). No entanto, apesar destes resultados expressivos, ainda são escassos na literatura científica investigações acerca dos atletas paralímpicos, mais especificamente dos paratletas das modalidades de atletismo e natação.

Na perspectiva do esporte competitivo em geral, é fundamental que a preparação dos atletas seja oriunda de uma atividade sistemática que visa promover adaptaçóes morfológicas, metabólicas e funcionais, as quais devem estar otimizadas na pré-temporada para os que os atletas consigam ter um desempenho ótimo na competição (MOREIRA et al., 2012).

No contexto específico das modalidades de atletismo e natação, os paratletas são submetidos a um calendário intenso que envolve múltiplas etapas competitivas durante o ano, ou seja, apresenta um curto período pré-competitivo e longa temporada competitiva. Dessa forma, a intensificação da carga de treinamento é uma estratégia comumente utilizada pelos treinadores e comissão técnica durante o período pré-competitivo, com o objetivo de alcançar níveis ótimos de desempenho no momento que antecede o período competitivo (PIRES; PIRES; FIGUEIRA JUNIOR, 2017).

A administração dessas variáveis de treino, em especial o volume, pode apresentar relação dose-resposta negativa entre nível de atividade física e predisposição ao risco de "supensação" (SAW; MANCINI; HUMPHRIES, 2016), "overtraining" (BORRESEN; LAMBERT, 2009), lesões e doenças (DREW; FINCH, 2016; SAW et al., 2016) e consequentemente uma menor percepção de qualidade de vida- QV (COSTA; SANTOS, 2002; VALOVICH et al., 2009; MARQUES; BRANDÁO, 2010).

Dessa forma, a mudança de perspectiva em torno do aspecto social, e a substituição do amadorismo pelo profissionalismo dos paratletas, sempre em busca constante por resultados, pode levar tanto ao desgaste físico quanto psicológico, o que pode influenciar a percepção do indivíduo sobre sua qualidade de vida (QV) (PARREIRAS, 2008).

Para isso, a utilização de instrumentos subjetivos que incluem a auto percepçáo e a auto avaliaçáo de todos os fatores envolvidos no esporte é considerada uma opção rápida e eficaz para a investigação do bem-estar (BOWLING, 2005). Alguns desses instrumentos de investigação, como o WHOQOL-BREF e o SF-36 têm sido utilizados para conhecer a QV em diversas populaçôes, incluindo atletas com deficiência.

De fato, Yazicioglu et al. (2012) compararam a QV em atletas com e sem deficiência utilizando o WHOQOL-BREF e constataram que os atletas com deficiência tiveram uma maior percepção e satisfação da QV. O estudo de Litchke et al. (2012) comparou os efeitos de 9 semanas de treinamento e a QV em atletas com deficiência e observou-se que o treinamento influenciou positivamente a percepção em alguns aspectos da QV como vitalidade e redução da dor corporal.

No entanto, apesar de vários estudos internacionais abordarem a QV em atletas sobre vários aspectos e populações, evidenciou-se uma escassez de estudos sobre esta temática no Brasil. O detalhamento dessa análise por questóes sócio-demográficas e o rendimento esportivo pode contribuir na identificação de aspectos mais críticos e grupos mais vulneráveis, o que, por sua vez, pode auxiliar na determinaçáo de planos de treinamento mais eficazes. Assim, este estudo teve como objetivo avaliar a percepçáo da qualidade de vida em atletas paralímpicos de atletismo e natação.

\section{Método}

\subsection{Participantes}

Foram avaliados 32 atletas paralímpicos brasileiros de ambos os gêneros, portadores de deficiência física, visual e intelectual, praticantes da modalidade atletismo $(n=22)$ e natação $(n=10)$. O estudo foi 
o de corte transversal e descritivo, realizado no período de maio a outubro de 2016, aplicado em equipes de atletismo e natação, localizadas no Brasil. A pesquisa foi aprovada pelo Comitê de Ética da Universidade Estácio de Sá sob registro CAAE:25927114.1.0000.5284 e todos os atletas assinaram o Termo de Consentimento Livre e Esclarecido concordando em participar voluntariamente da pesquisa. Os atletas foram entrevistados nas dependências de seus respectivos clubes, onde responderam aos questionários sócio-demográficos e WHOQOL-BREF para analisar a percepçáo de qualidade de vida.

\subsection{Procedimentos}

Para a coleta dos dados demográficos, um questionário foi elaborado pelos autores abrangendo questóes sociodemográficas (idade, gênero, escolaridade, caracterização do praticante, patrocínio, modalidade, grupo de prova, duração do treino e tempo de treinamento) com o objetivo de caracterizar a amostra. Para verificar a QV, foi utilizado o questionário WHOQOL-BREF que consta de 26 questóes, versão em português composto por 24 questốes abrangendo os domínios: físico, psicológico, relaçóes sociais e meio ambiente, além de duas questôes gerais (FLECK, 2000).

\subsection{Análise estatística}

Inicialmente, a normalidade dos dados foi verificada através do teste de Shapiro-Wilk, como os dados apresentaram distribuição não paramétrica, foi adotada a apresentaçáo descritiva em mediana (Md) e intervalo interquartílico (Q1; Q3). Para comparar a QV em função do gênero e do patrocínio, foi utilizado o teste de "U" de Mann-Whitney, e para comparar a QV entre o nível de escolaridade, foi utilizado o teste de Kruskal-Wallis. Ambos os testes consideraram $\mathrm{p}<0,05$ e foram obtidos através do pacote estatístico Statistical Package For The Social Sciences (SPSS) - IBM 22.0, devidamente registrado.

\section{Resultados e Discussão}

O presente estudo avaliou a percepção da qualidade de vida de atletas paralímpicos de atletismo e natação. Um grande número de trabalhos tem retratado que a prática de esportes é capaz de induzir melhoras tanto na perspectiva física como cognitiva, elevando a percepção da QV entre os atletas com deficiência (YAZICIOGLU et al., 2012; LITCHKE et al., 2012; FEITOSA et al., 2017).
Neste sentido, quanto à análise entre os domínios da QV entre os atletas paralímpicos (Tabela 1), observamos que o domínio ambiental foi o menor avaliado quando comparado com os outros domínios, corroborando com os achados de Esteves et al. (2015), que afirmaram em seu estudo que esse fato está relacionado à maioria dos atletas paralímpicos apresentarem baixo nível socioeconômico e esse contexto sociocultural, no qual o atleta está inserido, influencia diretamente na sua forma de pensar, sentir e agir.

Quando separamos o grupo de atletas paralímpicos por gênero (Tabela 2), não foram encontradas diferenças significativas. Tais resultados váo de encontro com os dados de Cucchiaro e Dalgalarrondo (2007), que verificaram que as mulheres atletas possuem as menores avaliações, sugerindo uma maior sensibilidade às condições ambientais precárias, afetando assim a QV. Um estudo comparou a QV entre homens e mulheres com deficiência, identificando-se que a qualidade e a satisfaçáo com a vida é mais bem avaliada em homens e mulheres por fatores como maior capacidade física e funcional, bem-estar, saúde em geral, apoio social, recursos financeiros e idade cronológica (TATE et al., 1997). Mas quando comparados entre atletas de alto rendimento, não houve diferenças significativas na QV, na questão gênero como demonstrado por Modoio et al. (2011).

É necessário destacar que em nosso estudo não foram constatadas diferenças significativas entre os paratletas que possuíam algum tipo de patrocínio esportivo público ou privado e os paratletas sem patrocínio (Tabela 3). Segundo a Organização Mundial de Saúde (OMS), fatores econômicos influenciam na percepção de QV (WORLD..., 2011). Contudo, nossos achados corroboram com os de Parreiras (2008) no qual o fator socioeconômico não interferiu na percepção de $\mathrm{QV}$ dos paratletas avaliados.

Em relação ao nível de escolaridade (Tabela 4), sabe-se que quanto maior o nível de instrução, melhor a percepção de qualidade de vida (NOCE; SIMIM; MELLO, 2009). Parreiras (2008) analisou a percepção de $\mathrm{QV}$ em atletas paralímpicos e

Tabela 1. Descrição da qualidade de vida de paratletas brasileiros.

\begin{tabular}{cc}
\hline DOMÍNIOS & Md $(\mathbf{Q} 1 ; \mathbf{Q} 3)$ \\
\hline Físico & $64,29(54,46 ; 95,35)$ \\
Psicológico & $72,91(62,50 ; 83,33)$ \\
Social & $75,00(50,00 ; 91,67)$ \\
Ambiental & $56,25(47,66 ; 64,84)$ \\
QV total & $66,07(56,72 ; 76,78)$ \\
\hline
\end{tabular}

$\mathrm{Md}=$ Mediana; $\mathrm{QV}=$ Qualidade de Vida. 
Tabela 2. Comparação da qualidade de vida em paratletas brasileiros em função do gênero.

\begin{tabular}{cccc}
\hline \multirow{2}{*}{ DOMÍNIOS } & \multicolumn{2}{c}{ Gênero } & \multirow{2}{*}{$\mathbf{p}$} \\
\cline { 2 - 3 } & Masculino & Feminino & \\
\cline { 2 - 3 } & Md (Q1; Q3) & Md (Q1; Q3) & 0,273 \\
Físico & $64,29(57,14 ; 77,67)$ & $57,14(47,32 ; 66,96)$ & 0,717 \\
Psicológico & $79,17(53,12 ; 83,33)$ & $68,75(63,54 ; 73,95)$ & 0,428 \\
Social & $62,50(50,00 ; 91,67)$ & $75,00(68,75 ; 93,75)$ & 0,983 \\
Ambiental & $56,25(47,66 ; 64,84)$ & $56,25(47,66 ; 64,06)$ & 0,881 \\
QV total & $68,15(56,34 ; 78,31)$ & $64,84(59,64 ; 66,77)$ & \\
\hline
\end{tabular}

$\mathrm{Md}=$ Mediana; $\mathrm{p}=$ Significância; $\mathrm{QV}=$ Qualidade de Vida.

Tabela 3. Comparação da qualidade de vida de paratletas brasileiros em função do patrocínio.

\begin{tabular}{|c|c|c|c|}
\hline \multirow{3}{*}{ DOMÍNIOS } & \multicolumn{2}{|c|}{ Patrocínio } & \multirow{3}{*}{$\mathrm{p}$} \\
\hline & Sim & Não & \\
\hline & Md (Q1; Q3) & Md (Q1; Q3) & \\
\hline Físico & $69,64(57,14 ; 75,00)$ & $60,71(45,53 ; 80,35)$ & 0,283 \\
\hline Psicológico & $77,08(65,62 ; 83,33)$ & $64,58(48,95 ; 84,37)$ & 0,338 \\
\hline Social & $70,83(58,33 ; 93,75)$ & $75,00(47,91 ; 91,67)$ & 0,587 \\
\hline Ambiental & $56,25(49,22 ; 63,28)$ & $54,69(43,75 ; 66,41)$ & 0,750 \\
\hline QV total & $67,87(59,66 ; 74,84)$ & $62,48(46,96 ; 78,75)$ & 0,301 \\
\hline
\end{tabular}

$\mathrm{Md}=$ Mediana; $\mathrm{p}=$ Significância; $\mathrm{QV}=$ Qualidade de Vida.

Tabela 4. Comparação da qualidade de vida de paratletas brasileiros em função do nível de escolaridade.

\begin{tabular}{cccccc}
\hline \multirow{2}{*}{ DOMíNIO } & \multicolumn{5}{c}{ Escolaridade } \\
\cline { 2 - 5 } & Fundamental I & Fundamental II & Ensino Médio & Ensino Superior & \multirow{2}{*}{ p $^{*}$} \\
\cline { 2 - 5 } & Md (Q1; Q3) & Md (Q1; Q3) & Md (Q1; Q3) & Md (Q1; Q3) & \\
\hline Físico & $53,57(42,86 ; 67,85)$ & $57,14(33,93 ; 85,71)$ & $64,29(62,50 ; 78,57)$ & $60,71(53,57 ; 85,71)$ & 0,329 \\
Psicológico & $45,83(33,33 ; 75,00)$ & $62,5(52,08 ; 85,41)$ & $79,17(64,58 ; 81,25)$ & $75,00(62,50 ; 87,50)$ & 0,184 \\
Social & $58,33(19,16 ; 91,66)$ & $50,00(37,5 ; 100,00)$ & $75,00(47,91 ; 91,67)$ & $66,67(54,16 ; 87,50)$ & 0,734 \\
Ambiental & $50,00(28,12 ; 54,69)^{1}$ & $50,00(46,87 ; 56,25)^{1}$ & $56,25(46,88 ; 67,19)$ & $62,50(57,81 ; 71,87)$ & $\mathbf{0 , 0 3 6 *}$ \\
QV & $56,29(43,19 ; 60,30)^{1,2}$ & $59,38(48,7 ; 73,51)$ & $67,93(61,18 ; 78,62)$ & $68,9(58,50 ; 81,45)$ & 0,094 \\
\hline
\end{tabular}

${ }^{*} \mathrm{p}<0,05 ;{ }^{1}$ diferença significativa com ensino superior; ${ }^{2}$ diferença significativa com ensino médio; $\mathrm{Md}=$ Mediana; $\mathrm{p}=$ Significância; $\mathrm{QV}=$ Qualidade de Vida.

verificou que o nível de escolaridade de atletas paralímpicos é maior em relação a pessoas com deficiência. Entretendo, nossos achados encontraram diferenças significativas quando comparamos os níveis de escolaridades, demonstrando que os atletas com níveis mais elevados apresentaram maiores percepçôes de QV nos domínios ambiental e de QV geral. Tais resultados são novos na literatura e podem ser explicados através dos dados do IBGE, 2010 , em que $23,4 \%$ dos entrevistados que se declararam portadores de algum tipo de deficiência, apresentaram baixo nível socioeconômico, tendo o predomínio econômico precário associado a baixa condição de escolaridade (INSTITUTO..., 2010; NIELLA, 2000).

Neste estudo pode-se observar algumas limitaçóes consideráveis. Evidentemente, torna-se limitado avaliar exclusivamente por meio de quantificaçôes um conceito intrinsecamente marcado pela subjetividade, como é o construto QV. É preciso ter em mente que os indicadores e índices medem sempre "aspectos" da QV. Neste sentido, os resultados observados no presente estudo precisam ser analisados com cautela, tendo em vista que a medida objetiva da QV foi mensurada de forma subjetiva através de questionário. No entanto, é importante ressaltar que o instrumento utilizado tem se apresentado como uma possibilidade de análise da QV prática e confiável, em adição, o WHOQOL-Bref é recomendado pela OMS para avaliação da QV (WHOQOL GROUP, 1995, 1998).

Além da complexibilidade para mensurar a QV, outras limitaçóes do presente estudo consistem no calendário esportivo, período de treinamento, competiçôes e lesôes, principalmente entre os atletas de níveis mais competitivos, bem como o tamanho da amostra. Por fim, esse estudo apresentou delineamento transversal e não foi possível estabelecer uma relação de causa e efeito entre o treinamento esportivo e a QV dos paratletas. 


\section{Considerações Finais}

Em suma, os resultados do presente estudo mostraram que atletas paralímpicos da modalidade atletismo e natação não apresentaram diferenças significativas na QV geral, gênero e patrocínio. Entretanto, quando comparamos os níveis de escolaridades, fundamental I e nível superior apresentaram diferenças nos domínios ambientais e de QV geral; comparando o ensino fundamental I com ensino médio, apenas na QV geral e comparando ensino fundamental II com ensino superior, apenas no domínio ambiental. Ademais, se sugere uma maior intervenção dos órgãos responsáveis em relação à segurança física, transporte e recursos financeiros (bolsas, financiamento de materiais e equipamentos) para essa população específica que tem contribuído para engrandecer o esporte brasileiro por meio de conquistas altamente significativas.

\section{Referências}

BARYAEV, A.; SHELKOV, O.; EVSEEV, S. XIII Paralympic games 2008 in Beijing: the analysis of results. In: ANNUAL CONGRESS OF THE EUROPEAN COLLEGE OF SPORT SCIENCE, 14., 2009, Noruega. Proceedings... Noruega: European College of Sport Science, 2009. p. 24-27.

BECKMAN, E. M. et al. Novel strength test battery to permit evidence-based paralympic classification. Medicine, Baltimore, v. 93, n. 4, p. e31, 2014. http://dx.doi. org/10.1097/MD.0000000000000031.

BORRESEN, J.; LAMBERT, M. I. The quantification of training load, the training response and the effect on performance. Sports Medicine, New Zealand, v. 39, n. 9, p. 779-795, 2009. http://dx.doi.org/10.2165/11317780000000000-00000.

BOWLING, A. Mode of questionnaire administration can have serious effects on data quality. Journal of Public Health, Oxford, v. 27, n. 3, p. 281-291, 2005. http:// dx.doi.org/10.1093/pubmed/fdi031.

COSTA, A. M.; SANTOS, S. S. Participação do Brasil nos Jogos Paraolímpicos de Sydney: apresentação e análise. Revista Brasileira de Medicina do Esporte, São Paulo, v. 8, n. 3, p. 70-76, 2002. http://dx.doi.org/10.1590/S151786922002000300002 .

CUCCHIARO, G.; DALGALARRONDO, P. Saúde mental e qualidade de vida em adolescentes: um estudo entre escolares em duas áreas urbanas contrastantes. Revista Brasileira de Psiquiatria, São Paulo, v. 29, n. 3, p. 213-221, 2007. http://dx.doi.org/10.1590/S151644462007000300005 .

DREW, M. K.; FINCH, C. F. The relationship between training load and injury, illness and soreness: a systematic and literature review. Sports Medicine, New Zealand, v.
46, n. 6, p. 861-883, 2016. http://dx.doi.org/10.1007/ s40279-015-0459-8.

ESTEVES, A. M. et al. Avaliação da qualidade de vida e do sono de atletas paralímpicos brasileiros. Revista Brasileira de Medicina do Esporte, São Paulo, v. 21, n. 1, p. 53-56, 2015.

FEITOSA, L. C. et al. Efeito do esporte adaptado na qualidade de vida e no perfil biopsicossocial de crianças e adolescentes com paralisia cerebral. Revista Paulista de Pediatria, São Paulo, v. 35, n. 4, p. 429-435, 2017. http:// dx.doi.org/10.1590/1984-0462/;2017;35;4;00001.

FLECK, M. P. A. O instrumento de avaliaçáo de qualidade de vida da Organização Mundial da Saúde (WHOQOL-100): características e perspectivas. Revista Ciência \& Saúde Coletiva, Rio de Janeiro, v. 5, n. 1, p. 33-38, 2000.

INSTITUTO BRASILEIRO DE GEOGRAFIA E ESTATÍSTICA - IBGE. Censo demográfico 2010. Rio de Janeiro: IBGE, 2010. Disponível em: <http://censo2010. ibge.gov.br/>. Acesso em: 22 ago. 2018.

INTERNATIONAL PARALYMPIC COMMITTEE - IPC. 50 years: remembering Rome. The Paralympian, [s.l.], n. 3, p. 6-9, 2018.

LITCHKE, L. G. et al. Effects of concurrent respiratory resistance training on health-related quality of life in wheelchair rugby athletes: a pilot study. Topics in Spinal Cord Injury Rehabilitation, St. Louis, v. 18, n. 3, p. 264272, 2012.

MARQUES, L. E.; BRANDÁO, M. R. F. Volume de treinamento, percepçáo subjetiva do esforço e estados de humor durante um macrociclo de treinamento. Revista Brasileira de Psicologia do Esporte, Brasília, v. 3, n. 1, p. 63-78, 2010.

MODOIO, V. B. et al. Negative addiction to exercise: are there differences between genders? Clinics, São Paulo, v. 66, n. 2, p. $255-260$, 2011. http://dx.doi.org/10.1590/ S1807-59322011000200013.

MOREIRA, A. et al. Monitoring internal load parameters during simulated and official basketball matches. Journal of Strength and Conditioning Research, Champaign, v. 26, n. 3, p. 861-866, 2012. http://dx.doi.org/10.1519/ JSC.0b013e31822645e9.

NIELLA, M. F. Familia y deficiencia mental. Salamanca: Amarú, 2000.

NOCE, F.; SIMIM, M. A. M.; MELLO, M. T. A percepção de qualidade de vida de pessoas portadoras de deficiência física pode ser influenciada pela prática de atividade física? Revista Brasileira de Medicina do Esporte, Brasília, v. 15, n. 3, p. 174-178, 2009. http://dx.doi.org/10.1590/S151786922009000300002 .

PARREIRAS, L. A. M. Análise dos fatores que influenciam a qualidade de vida de atletas paraolímpicos em ambientes de treinamento e competição. 2008. 96 f. Dissertação (Mestrado em Educaçáo Física) - Universidade Federal de Minas Gerais, Belo Horizonte, 2008. 
PIRES, G. P.; PIRES, K. C.; FIGUEIRA JUNIOR, A. J. Efeitos de 14 semanas de treinamento de força com periodização linear e ondulatória diária nas variáveis cinemáticas de jovens atletas de natação competitiva. Revista Brasileira de Ciências do Esporte, Brasília, v. 39, n. 3, p. 291-298, 2017. http://dx.doi.org/10.1016/j. rbce.2017.02.010.

SAW, J.; MANCINI, G. B. J.; HUMPHRIES, K. H. Contemporary review on spontaneous coronary artery dissection. Journal of the American College of Cardiology, New York, v. 68, n. 3, p. 297-312, 2016. http://dx.doi. org/10.1016/j.jacc.2016.05.034.

TATE, D. G. et al. Quality of life issues among women with physical disabilities or breast cancer. Archives of Physical Medicine and Rehabilitation, Philadelphia, v. 78, n. 12, p. S18-S25, 1997. http://dx.doi.org/10.1016/ S0003-9993(97)90217-3.

VALOVICH, T. C. et al. Recent injury and health-related quality of life in adolescent athletes. Journal of Athletic
Training, Dallas, v. 44, n. 6, p. 603-610, 2009. http:// dx.doi.org/10.4085/1062-6050-44.6.603.

WHOQOL GROUP. The world health organization quality of life assessment (WHOQOL). Position paper from the World Health Organization. SocSci Med, Oxford, v. 41, n. 10, p. 1403-1409, 1995. http://dx.doi.org/10.1016/02779536(95)00112-K.

WHOQOL GROUP. Development of the World Health Organization WHOQOL-BREF quality of life assessment. Psychological Medicine, London, v. 28, n. 3, p. 551-558, 1998. http://dx.doi.org/10.1017/S0033291798006667.

WORLD HEALTH ORGANIZATION - WHO. Global status report on communicable diseases 2010. Geneva, 2011.

YAZICIOGLU, K. et al. Influence of adapted sports on quality of life and life satisfaction in sport participants and non-sport participants with physical disabilities. Disability and Health Journal, New York, v. 5, n. 4, p. 249-253, 2012. http://dx.doi.org/10.1016/j.dhjo.2012.05.003.

\section{Contribuição dos Autores}

Gabriel Lucas Morais Freire foi responsável pela concepção do texto, organização das fontes, redação do texto e revisão final. Carla Thamires Laranjeira Granja foi responsável pela análise estatística, concepção do texto e revisão final. Vanthauze Marques Freire Torres foi responsável pela elaboração do projeto e redação do texto. Gustavo César de Vasconcelos foi responsável pela análise estatística. Milena Pedro de Morais foi responsável pela redação do texto e revisão final. Todos os autores aprovaram a versão final do texto. 